\title{
The Key Points on Deepening Poverty Alleviation and Elimination from Supply-side Structural Reform
}

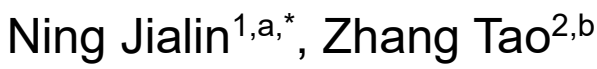 \\ ${ }^{1}$ Business School, Beijing Normal University, Haidian District, Beijing, China. \\ ${ }^{2}$ School of Economics and Resource Management, Beijing Normal University, Haidian District, \\ Beijing, China. \\ a18811338719@163.com, bzhangtaosyu@163.com. \\ ${ }^{*}$ Corresponding author
}

Keywords: Supply-side Structural Reform, Poverty Alleviation and Elimination, Condition, Approach.

\begin{abstract}
This paper explores the effectiveness of poverty alleviation and elimination from external management and endogenous development as the main line of supply-side structural reform, addresses the continuous new problems of poverty alleviation and elimination in new era including low quality poverty alleviation and elimination, short-term effect of poverty elimination and a series of structural new conflicts in poverty alleviation and elimination. On this basis, the measures shall be taken for support from the input side. The core and importance of the measures are to deepen the poverty alleviation and elimination on the main line of supply-side structural reform, so as to achieve breakthroughs in the major key points and directions including the structural reform, adjustment, optimization and upgrading, the improvement and guarantee of poverty elimination quality, the innovative driving and leading of poverty alleviation and elimination and the sustainable development of poverty alleviation and elimination.
\end{abstract}

\section{Introduction}

Poverty alleviation is the redistribution of social income. Income distribution concerns social justice.By means of poverty alleviation, the reasonable fair order of income distribution can be established. As the targeted poverty alleviation goes deeper, it has been transformed into a supply of public policies. In the new economic situation, the state proposes the "supply-side reform", which means to start with improving the supply quality and adopting the reform method to promote the structural adjustment, rectify the unreasonable factor allocation, expand the effective supply, improve the adaptability and flexibility of supply structure, improve the total factor productivity, so as to promote the sustainable and healthy development of economy and society. In this paper, the supply-side structural reform is taken as the main line. The thesis structure is as follows: first of all, to expound the current situation of poverty alleviation and elimination; secondly, to analyze and deepen the key points of poverty alleviation and elimination; lastly to make the conclusion and discussion.

\section{Current situation of poverty alleviation and elimination}

\subsection{To form the external management mechanism, highlight the crucial position of poverty elimination}

2016 is the first year to implement the 13th Five-Year Plan and the crucial top design of national poverty elimination. Obviously, China has obtained favorable results in poverty alleviation and elimination in 2016; the crucial poverty elimination has occupied an increasingly highlighted position in the overall work of the Party and nation. The situation that the whole party is mobilized to promote breakthroughs has been basically formed with various decisions, and deployments have been well implemented. The target poverty alleviation and elimination has gone down to the grass roots while the objective of annual poverty reduction has been well performed. It can be said that 
our mechanism of targeted poverty alleviation and elimination in the new era has been basically perfected, the new poverty elimination system has been basically established, the working mechanism of poverty alleviation and elimination has been strengthened and established ${ }^{[1]}$, the targeted identification mechanism has been completely implemented, the monitoring mechanism of poverty has been further improved and the appraisal mechanism has been stably carried out.

\subsection{Cultivate the endogenous development mechanism, realize the overall poverty elimination}

The endogenous mechanism of poverty alleviation and elimination is the fundamental dynamic mechanism for the Chinese mechanism of poverty alleviation and elimination. President Xi Jinping once pointed out that "Teach him how to fish and he eats fish for a lifetime. Education support is necessary in poverty alleviation. Enabling kids in depressed areas to receive good education is not only an important task for the poverty alleviation development but also an important approach to block the inter-generational transmission of poverty". As the poverty alleviation and elimination being carried forward, in addition to the formation of external management mechanism, the endogenous development mechanism of poverty alleviation and elimination has also been gradually cultivated and developed ${ }^{[3]}$. Mechanisms drawing the key attention of the nation, i.e. ability development of poverty alleviation through education and industry as well as poverty elimination through ideological and cultural promotion, have also been gradually formed and displaying certain effects.

\subsection{Analysis on the contradiction of supply-side structural reform of precision poverty alleviation}

During the process of poverty alleviation and elimination, we have also noticed the continuous new problems, confusions and conflicts brought forth by the poverty alleviation and elimination in new era. Indeed, the influences and potential hidden troubles are also expanded gradually, which is mainly reflected from the surface as follows: there are outstanding problems in works i.e. low quality of poverty alleviation, unsubstantial assist and support, inaccurate measures for poverty alleviation and fund supervision to be strengthened etc. It results in a series of new conflicts, including a series of structural new conflicts in poverty alleviation and elimination etc; low quality poverty alleviation and elimination and short-term effect of poverty elimination, influenced sustainable long-term effect of poverty elimination; what's more, the insufficient innovative concepts and behaviors in the ways and means of poverty elimination.

\section{Theoretical analysis and exploration based on the supply-side structural reform}

\subsection{Theory of poverty alleviation and elimination on the supply-side structure reform}

From the perspective of economics, the stable development of national economy is determined by the balance of supply and demand in market. The classical school stresses the function of supply-side, emphasizes the adjustment of economic structure and lays stress on improving the supply ability of national economy through the technical innovation and production efficiency. The theoretical analysis model is established on the basis of this theory.

In the current economic situation, the state has implemented the strategy of "targeted poverty alleviation and targeted poverty elimination". However the present phenomena including the unbalance of supply and demand in poverty alleviation, dislocation of supply and demand, inverse proportion between the input and output of poverty alleviation have turned the principal contradiction in poverty alleviation field from insufficient supply to the structural contradiction ${ }^{[2]}$. In 2017, it is necessary to lay a solid foundation, implement and refine the file creation, so as to achieve the dynamic management. The basic principle of strategy implementation according to classification shall be upheld while the effective measures shall be taken according to the causes of poverty. For places in extreme poverty, difficulty and importance, the super-normal measures shall be taken for support. 


\subsection{Deepen the structural reform and optimization of poverty alleviation and elimination}

The core of supply-side structural reform is the structural optimization and upgrading ${ }^{[3]}$. The key point of the supply-side structural reform in poverty alleviation and elimination is to solve the new structural problems and contradictions of poverty alleviation and elimination. To be specific: first, the regional structural contradictions of poverty alleviation and elimination. To be specific the inconsistent speed and level of poverty alleviation and elimination between the different areas and the different depressed counties. The counter flows and new differences between the depressed villages and non-depressed villages, the poverty-stricken families and non-poverty-stricken families. Second, the policy contradictions of poverty alleviation and elimination. The differences of poverty alleviation and elimination between different depressed villages and counties in terms of time and degree have resulted in the contradictions between the unity and difference of policy. The new structural contradictions between the funds and projects of poverty alleviation and elimination can mainly be reflected from the fact that there are project vacancies or the projects pay more attention to the short-term poverty alleviation projects despite the increased funds. Third, the new structural contradictions between social strengths and means of poverty alleviation and elimination. On the one hand, the structural contradictions between the social strength and local start to emerge. There emerges the divergences and conflicts between the social and local poverty alleviation strengths in terms of divided responsibility management, it's difficult for the social strength to play the promoting role for it which has been frozen by the local strength. On the other hand, the new structural contradictions between the ways and means of social security and poverty alleviation and elimination are increasingly outstanding.

\subsection{Improve the quality and level of poverty alleviation and elimination}

An important aspect of supply-side structural reform is the improvement of quality and level. For poverty alleviation and elimination, it is to improve the quality and level of targeted poverty alleviation and elimination. We believe the following major problems are important to be solved: first, it requires improve the quality of poverty alleviation through industry. On the one hand, efforts shall be strengthened to improve the quality of poverty alleviation through industry. On the other hand, the short-term behaviors which has resulted in the insufficient reserve strength for industrial development, low quality of poverty elimination and poor stability shall be overcome. Second, it requires improve the quality of targeted poverty alleviation and elimination, including improving the professional level and quality of the support teams on the original basis. And the poverty alleviation projects must be the most effective, most popular among peasants and most practical to improve the peasants. Third, it requires shift the attention from focusing only on the income-based poverty to paying more attention to the three guarantees, which means, the social security, infrastructure and public service must be improved synchronously.

\subsection{Innovation and cultivation of dynamic mechanism of poverty alleviation and elimination}

To innovate the development power is one of the important structural contents at the supply-side as well as the breakthrough for the structural reform at the supply-side of Chinses poverty alleviation and elimination in the new era. The current poverty alleviation and elimination is exogenous dynamic mechanism driven powerfully by the poverty alleviation and elimination under the governmental leadership. In the current situation, the governmental powerful pulling and pushing are inseparable. On the other hand, the concentrated expression of Chinses political and system superiority is to collect resources, funds and talents within short time to deal with important matters. However, under such powerful external strength, the rapid collection within short time will definitely lead to some disadvantages including the weakened, degraded, deactivated and even depraved internal dynamic mechanism of the depressed villages and poverty-stricken families and so on. By this token, Cultivation of the internal power mechanism of poverty alleviation and elimination like knowledge education and traditional culture is also one of the key tasks for supply-side structural reform of poverty alleviation and elimination. The proportion of soft investment shall be increased at the same time of hard investment, so as to improve the training on 
knowledge i.e. ideological concept and cultural consciousness in cultivating self-independence, improve the heritage of the traditional culture and concept for poverty alleviation through hard work.

\subsection{Enhancement of sustainable functions of poverty alleviation and elimination}

Poverty alleviation and elimination is a long-term task. It is a goal to solve the rural poverty by 2020 under the existing standards which means to eliminate the absolute poverty; yet poverty reduction will be an eternal topic. It is to focus on the future and figure out whether the poverty alleviation and elimination is of sustainable functions. So how to start with the current poverty alleviation and elimination to form the sustainable mechanism of poverty alleviation and elimination? We believe that in addition to poverty alleviation and elimination through education, the green poverty alleviation and elimination is a strategic choice that cannot be ignored for the Chinese poverty alleviation and elimination in a period of time to come and even after 2020. Green and biological poverty alleviation and elimination has been put into practice from the new concept ${ }^{[4]}$; the important task of the cultivation and growth of the sustainable functions for the Chinese poverty alleviation and elimination is to explore the green poverty alleviation and elimination, which is another key point for the supply-side structural reform of poverty alleviation and elimination.

\section{References:}

[1] J.Hu, Deepening the supply - side reform and improving the efficiency of precision poverty alleviation, Economic Daily, 2016-06-10.

[2] Y.M.Zhang, Z.S.Tang and Z.M.Hu, Policy Suggestions on Promoting the Innovation of Poverty Alleviation Pattern in "Central Capital Poverty Zone", Economy Forum, iss.4, 2014.

[3] Y.M.Zhang, Exploring supply-side reform to help precision poverty alleviation, Enterprise Economy, vol.441, iss.5, pp.5-11, 2017.

[4] Ecological Civilization Construction and Anti - poverty Guiyang Consensus, http://news.china.com.cn/cndg/2016-07/10/content_38849009.htm, 2016-07-09. 